\title{
BOUNDS FOR THE ASYMPTOTIC GROWTH RATE OF AN AGE-DEPENDENT BRANGHING PROCESS
}

\author{
P. J. BROCKWELL ${ }^{1}$
}

(Received 22 April 1968)

Let $M(t)$ denote the mean population size at time $t$ (conditional on a single ancestor of age zero at time zero) of a branching process in which the distribution of the lifetime $T$ of an individual is given by $\operatorname{Pr}\{T \leqq t\}=G(t)$, and in which each individual gives rise (at death) to an expected number $A$ of offspring $(1<A<\infty)$. Then it is well-known (Harris [1], p. 143) that, provided $G(0+)-G(0-)=0$ and $G$ is not a lattice distribution, $M(t)$ is given asymptotically by

$$
M(t) \sim \frac{A-1}{c A^{2} \int t e^{-c t} d G(t)} e^{c t}, \quad t \rightarrow \infty,
$$

where $c$ is the unique positive value of $p$ satisfying the equation

$$
\int e^{-p t} d G(t)=A^{-1} \text {. }
$$

In many biological problems the distribution function $G$ is not known precisely and it is of interest to find bounds for the asymptotic growth rate $c$ (sometimes known as the Malthusian parameter for the population), given only that

$$
\int t d G(t)=m_{1}
$$

or

(b)

$$
\int t d G(t)=m_{1} \text { and } \int t^{2} d G(t)=m_{2},
$$

where $m_{1}, m_{2}<\infty$.

In this note we shall find the best possible bounds for $c$ under these conditions and, in the course of the derivation, determine the functions (defined for all real non-negative values of $p$ ) $\sup _{F \in \mathscr{F}} \Phi(F, p)$ and $\inf _{F \in \mathscr{F}} \Phi(F, p)$, where $\Phi(F, p)=\int e^{-p t} d F(t)$ and $\mathscr{F}$ represents one or other of the classes of probability distribution functions:

1 Work performed under the auspices of the United States Atomic Energy Commission. 


$$
\begin{aligned}
\mathscr{F}\left(m_{1}\right) & =\left\{F: F(0-)=0, \int t d F(t)=m_{1}\right\}, \\
\mathscr{F}\left(m_{1}, m_{2}\right) & =\left\{F: F(0-)=0, \int t d F(t)=m_{1}, \int t^{2} d F(t)=m_{2}\right\} .
\end{aligned}
$$

Bounding techniques for branching processes have been used previously by Heathcote and Seneta [2], Senate [3] and Brook [4]. Lemmas 2 and 3 below were used by Brook to obtain an upper bound for the extinction probability.

Before deriving the results, which are given as a series of lemmas, we note that if $F$ is the distribution function of a proper non-zero nonnegative random variable and

$$
\Phi_{n}(F, p)=\int t^{n} e^{-p t} d F(t), \quad n=0,1,2, \cdots,
$$

then it is well-known that if $\Phi_{n}(F, 0)<\infty, \log \Phi_{n}(F, p)$ is strictly decreasing and convex for $p \geqq 0$.

It will be assumed throughout that $m_{1}>0$ since Lemmas $1-3$ are trivial if $m_{1}=0$.

LEMMA 1.

$$
\inf _{F \in \mathscr{F}\left(m_{1}\right)} \Phi(F, p)=\inf _{F \in \mathscr{F}\left(m_{1}, m_{\mathbf{2}}\right)} \Phi(F, p)=e^{-m_{1} p}, \quad 0 \leqq p<\infty .
$$

Proof. (i) We first show that $e^{-m_{1} p} \leqq \Phi(F, p)$ for all $F \in \mathscr{F}\left(m_{1}\right)$. Denote by $D_{p}$ the operator $d / d p$. Then since $\log \Phi(F, p)=0$ at $p=0$ and $D_{p} \log \Phi(F, p)=-m_{1}$ at $p=0$ it follows from the convexity of $\log \Phi(F, p)$ that $\log \Phi(F, p) \geqq-m_{1} p$ for all $p \geqq 0$.

(ii) By choosing $\alpha$ sufficiently small in the example

$$
F(t)=\left\{\begin{array}{l}
0, t<m_{1}-\sigma[\alpha /(1-\alpha)]^{\frac{1}{2}}, \\
1-\alpha, m_{1}-\sigma[\alpha /(1-\alpha)]^{\frac{1}{2}} \leqq t<m_{1}+\sigma[(1-\alpha) / \alpha]^{\frac{1}{2}}, \\
1, t \geqq m_{1}+\sigma[(1-\alpha) / \alpha]^{\frac{1}{2}},
\end{array}\right.
$$

(where $\sigma=\left(m_{2}-m_{1}^{2}\right)^{\frac{1}{2}}$ ) we see that for any given non-negative $p$ and positive $\varepsilon$ there exists $F \in \mathscr{F}\left(m_{1}, m_{2}\right)$ such that $\Phi(F, p)-e^{-m_{1} p}<\varepsilon$.

Remark. The example given in (ii) also shows that the infima are unchanged when taken over the subclass of $\mathscr{F}\left(m_{1}, m_{2}\right)$ in which $F(0+)-F(0-)=0$ and $F$ is a non-lattice distribution.

LEMMa 2.

$$
\sup _{F \in \mathscr{F}\left(m_{1}\right)} \Phi(F, p)=1, \quad 0 \leqq p<\infty .
$$

Proof. We need only show that for any given non-negative $p$ and positive $\varepsilon$ there exists $F \in \mathscr{F}\left(m_{1}\right)$ such that $1-\Phi(F, p)<\varepsilon$. Such an $F$ is obtained by choosing $\alpha$ sufficiently small in the following example: 


$$
F(t)= \begin{cases}0, & t<\alpha m_{1}, \\ 1-\alpha, & \alpha m_{1} \leqq t<\alpha^{-1} m_{1}\left(1-\alpha+\alpha^{2}\right), \\ 1, & t \geqq \alpha^{-1} m_{1}\left(1-\alpha+\alpha^{2}\right) .\end{cases}
$$

LEMMA 3.

$$
\sup _{F \in\left(m_{1}, m_{2}\right)} \Phi(F, p)=1-\frac{m_{1}^{2}}{m_{2}}+\frac{m_{1}^{2}}{m_{2}} \exp \left(-\frac{m_{2} p}{m_{1}}\right), \quad 0 \leqq p<\infty .
$$

Proof. (i) We first establish the inequality,

$$
\chi(F, p) \equiv \Phi(F, p)-1+\frac{m_{1}^{2}}{m_{2}}-\frac{m_{1}^{2}}{m_{2}} \exp \left(-\frac{m_{2} p}{m_{1}}\right) \leqq 0 .
$$

Since at $p=0$ there is equality in (3) it will be sufficient to show that $D_{p} \chi(F, p) \leqq 0$ for all non-negative $p$, or equivalently that

$$
\rho(F, p) \equiv \log \Phi_{1}(F, p)-\log m_{1}+m_{2} p / m_{1} \geqq 0 .
$$

Since $\rho(F, p)=0$ at $p=0$ and $D_{p} \rho(F, p)=0$ at $p=0$ it follows from the convexity of $\rho(F, p)$ that $\rho(F, p) \geqq 0$ for all $p \geqq 0$. This establishes the inequality (3).

(ii) If $m_{2}=m_{1}^{2}$ the assertion of the lemma is trivial since in this case $\sup _{F \in \mathscr{F}\left(m_{1}, m_{3}\right)} \Phi(F, p)=\exp \left(-m_{1} p\right)$. If $m_{2}>m_{1}^{2}$ then by choosing $\alpha$ sufficiently small $(\alpha>0)$ in the example,

$$
F(t)=\left\{\begin{array}{cc}
0, & t<m_{1}-\sigma\left[\left(m_{1}^{2}-m_{2} \alpha\right)\left(\sigma^{2}+m_{2} \alpha\right)^{-1}\right]^{\frac{1}{2}}, \\
1-\frac{m_{1}^{2}}{m_{2}}+\alpha, & m_{1}-\sigma\left[\left(m_{1}^{2}-m_{2} \alpha\right)\left(\sigma^{2}+m_{2} \alpha\right)^{-1}\right]^{\frac{1}{2}} \\
& \leqq t<m_{1}+\sigma\left[\left(\sigma^{2}+m_{2} \alpha\right)\left(m_{1}^{2}-m_{2} \alpha\right)^{-1}\right]^{\frac{1}{2}}, \\
1, & t \geqq m_{1}+\sigma\left[\left(\sigma^{2}+m_{2} \alpha\right)\left(m_{1}^{2}-m_{2} \alpha\right)^{-1}\right]^{\frac{1}{2}},
\end{array}\right.
$$

(where $\sigma=\left(m_{2}-m_{1}^{2}\right)^{\frac{1}{2}}$ ) we see that for any given non-negative $p$ and positive $\varepsilon$ there exists $F \in \mathscr{F}\left(m_{1}, m_{2}\right)$ such that

$$
1-\frac{m_{1}^{2}}{m_{2}}+\frac{m_{1}^{2}}{m_{2}} \exp \left(-\frac{m_{2} p}{m_{1}}\right)-\Phi(F, p)<\varepsilon .
$$

REMARK. The examples given in the proofs of Lemmas 2 and 3 show that the suprema are unchanged when the further restrictions are imposed that $F(0+)-F(0-)=0$ and that $F$ be a non-lattice distribution.

LEMMA 4. If $A>1, G(0-)=G(0+)=0, G$ is a non-lattice distribution, and $c(G)$ is the unique positive root of equation (2), then 


$$
\begin{aligned}
& \inf _{G \in \mathscr{F}\left(m_{1}\right)} c(G)=\frac{\log A}{m_{1}}, \sup _{G \in \mathscr{F}\left(m_{1}\right)} c(G)=\infty \\
& \inf _{G \in \mathscr{F}\left(m_{1}, m_{2}\right)} c(G)=\frac{\log A}{m_{1}}, \sup _{G \in \mathscr{F}\left(m_{1}, m_{2}\right)} c(G)=\left\{\begin{aligned}
\frac{m_{1}}{m_{2}} \log \frac{m_{1}^{2} A}{m_{1}^{2} A-m_{2}(A-1)} \\
\text { if } m_{1}^{2} A>m_{2}(A-1), \\
\infty \quad \text { if } m_{1}^{2} A \leqq m_{2}(A-1) .
\end{aligned}\right.
\end{aligned}
$$

Proof. If $G \in \mathscr{F}\left(m_{1}\right)$ satisfies the conditions of the lemma then we know from Lemma 1 that $\Phi(G, p) \geqq \exp \left(-m_{1} p\right)$, and in particular $\Phi(G, c(G))=A^{-1} \geqq \exp \left[-m_{1} c(G)\right]$. Hence $c(G) \geqq m_{1}^{-1} \log A$. Furthermore given any $\varepsilon>0$ it follows from Lemma 1 , since $\exp \left(-m_{1} p\right)<A^{-1}$ if $p=m_{1}^{-1} \log A+\varepsilon$, that there exists $G \in \mathscr{F}\left(m_{1}, m_{2}\right)$ satisfying the conditions of Lemma 4 such that $\Phi\left(G, m_{1}^{-1} \log A+\varepsilon\right)<A^{-1}$. Since $\Phi(G, p)$ is a decreasing function of $p$ this inequality implies that $c(G)<m^{-1} \log A+\varepsilon$. This establishes the infima as given in the statement of the lemma. The suprema are established in an analogous way from Lemmas 2 and 3.

It is interesting to observe that specification of only the mean of $F$ gives no finite upper bound for $c$. Specification of the second moment as well as the mean gives an upper bound for $c$ only if the coefficient of variation is sufficiently small (i.e., only if $\left.m_{1}^{-1}\left(m_{2}-m_{1}^{2}\right)^{\frac{1}{2}}<(A-1)^{-\frac{1}{2}}\right)$. A large coefficient of variation allows the probability of a lifetime near zero to become too great for $c$ to be bounded above.

In terms of a specified mean, $m_{1}$, and coefficient of variation $v$, Lemma 4 gives

$$
\log A \leqq m_{1} c \leqq \frac{1}{\left(1+v^{2}\right)} \log \frac{A}{1-(A-1) v^{2}}
$$

For reasonably small values of $v$ (as frequently occur in biological problems) these bounds are rather close. For example in the particular case $A=\mathbf{2}$, we obtain the following bounds for various values of $v$ :

$$
\begin{aligned}
& v=0.2, \quad 0.693 \leqq m_{1} c \leqq 0.706 ; \\
& v=0.4, \quad 0.693 \leqq m_{1} c \leqq 0.748 \text {; } \\
& v=0.6, \quad 0.693 \leqq m_{1} c \leqq 0.838 \text {; } \\
& v=0.8, \quad 0.693 \leqq m_{1} c \leqq 1.046 \text {; } \\
& v=1.0, \quad 0.693 \leqq m_{1} c \leqq \infty \text {. }
\end{aligned}
$$

We note finally that for given $A$ and $m_{1}$ the least upper bound for $c$ increases monotonically to $\infty$ as $v$ increases from zero to $(A-1)^{-\frac{1}{2}}$. Consequently if we specify that the mean lifetime be $m_{1}$ and that the coefficient of variation satisfy the inequality $v \leqq v_{0}<(A-1)^{-\frac{1}{2}}$, then the best bounds which can be given for $c$ are obtained from (4) on setting $v=v_{0}$. 


\section{Acknowledgement}

I am indebted to Dr. E. Trucco who raised this problem in connection with its biological applications and to the referee for several helpful comments.

\section{References}

[1] T. E. Harris, The theory of branching processes (Springer-Verlag, Berlin, 1963).

[2] C. R. Heathcote and E. Seneta, 'Inequalities for branching processes', J. Appl. Prob., 3 (1966), 261-67; Correction: 4 (1967), 215.

[3] E. Seneta, 'On the transient behaviour of a Poisson branching process', J. Austral. Math. Soc., 7 (1967), $465-80$.

[4] D. Brook, 'Bounds for moment generating functions and for extinction probabilities', J. Appl. Prob., 3 (1966), 171-178.

Argonne National Laboratory

Argonne, Illinois 